\title{
OPTIMIZATION OF THE NLC FINAL FOCUS SYSTEM *
}

\author{
F. Zimmermann, R. Helm, J. Irwin, Stanford Linear Accelerator Center, Stanford University, \\ Stanford, CA 94309 USA
}

\begin{abstract}
An optimization scheme for final focus systems is discussed and applied to the NLC design. The optical functions at the defocusing sextupoles, the sextupole strength, and the length of the system must obey eight conditions that are imposed by the spot size increase due to higher-order aberrations, the effects of synchrotron radiation in the bending magnets, power supply ripple, magnet vibration tolerances, and the estimated orbit stability at the sextupoles. These eight conditions determine the minimum optimum length of the system. The NLC final focus design was shortened to this optimum.
\end{abstract}

\section{INTRODUCTION}

In this report, an optimization scheme for final focus systems is proposed. The spot size increase by higher-order aberrations, synchrotron radiation effects, and the tolerances on power supply ripple, mechanical vibrations, and orbit stability depend on the length of the final focus system. This dependence results in scaling laws that are discussed in the next section. To evaluate these for the NLC final focus system, the values of certain length-independent parameters have been extracted from a preliminary, not optimized final-focus design. Based on these values, the optimum choice for dispersion and beta functions at the Y-sextupoles, for the sextupole strength and the length of the bend section are found. We roughly follow the analysis presented in Ref. [1]. However, the final optimization procedure is different.

\section{SCALING LAWS}

\section{A. A General Telescope}

We assume that the final focus system contains a horizontal and a vertical chromatic correction section (CCX and CCY) which are separated by a beta-exchange module (BX). Considering only quadrupole magnets of strength $k_{i}$, the Hamiltonian for either one of these three modules may be written

$$
H=-\frac{\delta}{2} \sum k_{i}\left(x_{i}+\eta_{i} \delta\right)^{2}+\frac{\delta}{2} \sum k_{i} y_{i}^{2} .
$$

We now transform to the starting point of the module using the sine- and cosine-like trajectories, $x_{i}=c_{i}^{x} x_{1}+s_{i}^{x} x_{1}^{\prime}$, $y_{i}=c_{i}^{y} y_{1}+s_{i}^{y} y_{1}^{\prime}$, to define the chromatic coefficients

$$
c^{x, y} \equiv \sum k_{i} c_{i}^{x, y 2}, \quad c^{x, y^{\prime}} \equiv 2 \sum k_{i} c_{i}^{x, y} s_{i}^{x, y},
$$

*Work supported by Department of Energy contract DE-AC0376 SF00515.

$$
\begin{gathered}
c^{x, y^{\prime \prime}} \equiv \sum k_{i} s_{i}^{x, y 2}, \\
d^{x} \equiv \sum k_{i} \eta_{i} c_{i}^{x},
\end{gathered}
$$

and get

$$
\begin{aligned}
H= & -\frac{\delta}{2}\left[c^{x} x_{1}^{2}+c^{x^{\prime}} x_{1} x_{1}^{\prime}+c^{x^{\prime \prime}} x_{1}^{\prime 2}+\delta d^{x} x_{1}\right. \\
& \left.+\delta d^{x^{\prime}} x_{1}^{\prime}\right]+\frac{\delta}{2}\left[c^{y} y_{1}^{2}+c^{y^{\prime}} y_{1} y_{1}^{\prime}+c^{y^{\prime \prime}} y_{1}^{\prime 2}\right]
\end{aligned}
$$

By symmetry, $c^{x^{\prime}}=d^{x}=c^{y^{\prime}}=0$ for CCX and CCY, and we assume the same for the BX. The $\beta$-dependence is then extracted from the coordinates, $x_{1}=\sqrt{\beta_{1}^{x}} x^{\prime}$, and $x_{1}^{\prime}=$ $\frac{x}{\sqrt{\beta_{1}^{x}}}, y_{1}=\sqrt{\beta_{1}^{y}} y^{\prime}, y_{1}^{\prime}=\frac{y}{\sqrt{\beta_{1}^{y}}}$, where $x, x^{\prime}, y, y^{\prime}$ are now normalized IP-coordinates, and length-independent chromatic coefficients are introduced, $d^{x^{\prime}}=\hat{d}^{x^{\prime}} L, c^{x, y}=c_{\bar{L}}$, $c^{x^{\prime}, y^{\prime}}=\hat{c}^{x^{\prime}, y^{\prime}}, c^{x^{\prime \prime}, y^{\prime \prime}}=L \hat{c}^{x^{\prime \prime}}, y^{\prime \prime}$. The Hamiltonian (3) is then written

$$
\begin{aligned}
H= & -\frac{\delta}{2}\left[\hat{c}^{x} \frac{\beta_{1}^{x}}{L} x^{\prime 2}+\hat{c}^{x^{\prime \prime}} \frac{L}{\beta_{1}^{x}} x^{2}-\hat{c}^{y} \frac{\beta_{1}^{y}}{L} y^{\prime 2}\right. \\
& \left.-\hat{c}^{y^{\prime \prime}} \frac{L}{\beta_{1}^{y}} y^{2}+2 \delta L \hat{d}^{x^{\prime}} \frac{1}{\sqrt{\beta_{1}^{x}}} x\right] .
\end{aligned}
$$

Finally, the dispersion at the $y$-sextupoles is converted into a length-independent parameter $\bar{r}_{12}$ via

$$
\eta=\theta_{B} L \bar{r}_{12},
$$

where $\theta_{B}$ is the bend angle of the last bending section at the end of the CCY, and $L$ the total length of the final focus system.

\section{B. The $C C X$ and the $B X$}

We rewrite Eq. (4) for the CCX in the form

$$
e^{-H_{C X}} \approx e^{-\frac{1}{2} F\left(x^{\prime}, y^{\prime}\right)} e^{-G(x, y)} e^{-\frac{1}{2} F\left(x^{\prime}, y^{\prime}\right)},
$$

where

$$
F\left(x^{\prime}, y^{\prime}\right)=-\frac{\delta}{2} \xi_{x}^{C X} x^{\prime 2}+\frac{\delta}{2} \xi_{y}^{C X} y^{\prime 2}
$$

$$
G(x, y)=-\frac{\delta}{2}\left[\hat{c}^{x^{\prime \prime}} \frac{L}{\beta_{F}^{x}} x^{2}-\frac{\delta}{2} \hat{c}^{\prime \prime} \frac{L}{\beta_{F}^{y}} y^{2}+2 \delta^{2} \hat{d}^{x^{\prime}} \frac{L}{\sqrt{\beta_{F}^{x}}} x\right],
$$

and we have introduced the horizontal and vertical chromaticities $\xi_{x}^{C X} \equiv \hat{c}^{x} \frac{\beta_{F}^{x}}{L}, \xi_{y}^{C X} \equiv \hat{c}^{y} \frac{\beta_{F}^{3}}{L}$. The Hamiltonians of the two $X$-sextupoles at the beginning and the end of the CCX, denoted by 1 and 2 , read

$$
\begin{aligned}
& H_{1}=\tilde{H}\left(x_{F}+\eta_{F} \delta, y_{F}\right) \\
& H_{2}=-\tilde{H}\left(x_{F}+\eta_{F} \delta, y_{F}\right)+k_{F} \eta_{F} \delta\left(x_{F}^{2}-y_{F}^{2}\right)
\end{aligned}
$$


respectively, where $\tilde{H}$ is the usual sextupole-Hamiltonian

$$
\tilde{H}(x, y) \equiv \frac{1}{3 !} k_{F}\left(x^{3}-3 x y^{2}\right),
$$

the term $k_{F}$ denotes the integrated sextupole strength and $x_{F} \equiv \sqrt{\beta_{F}^{x}} x^{\prime}$, and $y_{F} \equiv \sqrt{\beta_{F}^{y}} y^{\prime}$ are the coordinates at the first $\mathrm{X}$-sextupole. Now the total CCX, including sextupoles, is

$$
e^{-H_{1}} e^{-\frac{1}{2} F\left(x^{\prime}, y^{\prime}\right)} e^{-G(x, y)} e^{-\frac{1}{2} F\left(x^{\prime} \cdot y^{\prime}\right)} e^{-H_{2}}=e^{-G_{R}^{C X}} e^{-H_{R}^{C X}}
$$

where

$$
G_{R}^{C C X} \equiv G\left(x+\frac{\partial}{\partial x^{\prime}}\left(H_{1}+F\right), y+\frac{\partial}{\partial y^{\prime}}\left(H_{1}+F\right)\right)
$$

and

$$
H_{R}^{C C X} \equiv F^{C C X}+k_{F} \eta_{F} \delta\left(x_{F}^{2}-y_{F}^{2}\right) .
$$

In much the same manner the chromaticity can be propagated through the $\mathrm{BX}$ to give

$$
H_{R}^{B X}=F^{B X}+F^{C C X}+k_{F} \eta_{F} \delta\left(x_{F}^{2}-y_{F}^{2}\right)
$$

and a term $C_{R}^{B X}$ analogous to $C_{R}^{C C X}$. The generators $G_{R}^{C C X}$ and $G_{R}^{B X}$ contain fourth- and fifth-order terms, which have to be small.

\section{The $C C Y$}

The Hamiltonian (14) to be carried through the CCY is of the form

$$
H \approx-\frac{\delta}{2} \xi_{x}^{(3)} x^{2}+\frac{\delta}{2} \xi_{y}^{(3)} y^{\prime 2}+\ldots
$$

where

$$
\xi_{x, y}^{(3)} \equiv \xi_{x, y}^{C X}+\xi_{x, y}^{B X}+\frac{1}{2} \xi_{x, y}^{C Y}-2 k_{F} \eta_{F} \beta_{F}^{x, y}-k_{D} \eta_{D} \beta_{D}^{x, y},
$$

and it interacts with a term analogous to $G(x, y)$ above,

$$
G^{C Y}(x, y)=-\frac{\delta}{2} \hat{c}_{C Y}^{x^{\prime \prime}} \frac{L}{\beta_{D}^{x}} x^{2}-\delta^{2} L \hat{d}_{C Y}^{x^{\prime}} \frac{1}{\sqrt{\beta_{D}^{x}}} x+\ldots
$$

The largest aberrations generated by the kick in $x$ is a $\delta^{3}$-dispersion that could, at least in principle, be canceled downstream, and a $\delta^{3}$-chromaticity, from which

$$
\beta_{D}^{x} \geq \stackrel{\sqrt{15}}{ } \Delta_{x} \delta^{3} \xi_{x}^{(3)}{ }^{2} \hat{c}_{C Y}^{\prime \prime} L
$$

where $\Delta_{x} \approx 1$ denotes the maximum tolerable relative increase of the horizontal spot size. Similarly, the $y$-kicks generate a third-order vertical chromaticity and a $x^{2} y^{\prime 2} \delta$ term, giving rise to the two conditions

$$
\begin{gathered}
\sqrt{15} \delta^{3} \hat{c}_{C Y}^{y^{\prime \prime}} \frac{L}{\beta_{D}^{y}} \xi_{y}^{(3) 2} \leq \Delta_{y}, \\
\sqrt{3} \delta \hat{c}_{C Y}^{y^{\prime \prime}} k_{D}^{2} L \beta_{D}^{x} \beta_{D}^{y} \epsilon_{x} \leq \Delta_{y},
\end{gathered}
$$

where $\Delta_{y} \approx 1 / \sqrt{2}$ (see Section F).

\section{Synchrotron Radiation and Chromaticity}

The beam size increase, due to the additional energy spread $\delta_{\text {rms }}$, induced by synchrotron radiation inside and behind the CCY and to the uncompensated doublet chromaticity, $\xi_{y}^{F D} \approx 2 \xi_{y}^{(3)}$, has to be small:

$$
2 \delta_{r m s} \xi_{y}^{(3)}<\Delta_{E}
$$

where $\Delta_{E} \approx 1$ denotes the maximum tolerable relative increase of the vertical spot size due to synchrotron radiation (see later), and the energy spread $\delta_{r m s}$ is [3]

$$
\delta_{r m s}^{2} \approx 2 \frac{55}{24 \sqrt{3}} \frac{1}{2 \pi} r_{e} \lambda_{e} \gamma^{5} \frac{\theta_{B}^{3}}{L_{B}^{2}} .
$$

Here, $L_{B}$ denotes the length of the last bending section behind the CCY, and the factor 2 accounts for the contributions from bending magnets in the center of the CCY. The length of the central section is more than two times $L_{B}$, but in this case about half the doublet chromaticity is compensated by the final sextupole. Combining Eqs. (5), (20) and (21) and using $\alpha \equiv \frac{L}{L_{B}}$ we find

$$
\eta_{D} \leq \bar{r}_{12}\left(\frac{\Delta_{E}}{2 \xi_{y}^{(3)}}\right)^{\frac{2}{3}}\left(\frac{L^{5} 2 \pi}{\left(\frac{55}{24 \sqrt{3}}\right) r_{e} \lambda_{e} \gamma^{5} \alpha^{2}}\right)^{\frac{1}{3}} .
$$

The overall chromaticity balance reads approximately

$$
\eta_{D} k_{D} \beta_{D}^{y} \approx \xi_{y}^{(3)}+\frac{b}{2} \hat{c}_{C Y}^{y} \frac{\beta_{D}^{y}}{L}
$$

where $b \approx 2$.

\section{E. Long-Sextupole Effect}

A long sextupole generates octupole-like aberrations [2], which impose a limit on the tolerable sextupole length. For two sextupoles separated by a $-I$, these aberrations are described by the Hamiltonian

$$
H_{l s}=\frac{k_{D}^{2} l_{D}}{24}\left(\beta_{D}^{x}{ }^{2} x^{4}+2 \beta_{D}^{x} \beta_{D}^{y} x^{\prime 2} y^{\prime 2}+\beta_{D}^{y}{ }^{2} y^{\prime 4}\right),
$$

where $l_{D}$ denotes the sextupole length. From the resulting increase of the vertical spot size, we deduce

$$
\frac{k_{D}^{2} l_{D}}{6}\left(\sqrt{3} \beta_{D}^{x} \beta_{D}^{y} \epsilon_{x}+\sqrt{15} \beta_{S D}^{y} \epsilon_{y}\right) \leq \Delta_{y} .
$$

Decomposing the integrated sextupole strength as $k_{D}=$ $l_{D} \tilde{k}_{D}$, it follows that

$$
l_{D} \leq\left(\frac{6 \Delta_{y}}{\tilde{k}_{D}^{2}\left(\sqrt{3} \beta_{D}^{x} \beta_{D}^{y} \epsilon_{x}+\sqrt{15} \beta_{D}^{y}{ }^{2} \epsilon_{y}\right)}\right)^{\frac{1}{3}}
$$

Assuming a pole tip field of $0.5 \mathrm{~T}$ at a radius of $5 \mathrm{~mm}$, the maximum value of $\tilde{k}_{D}$ is about

$$
\tilde{k}_{D, \max } \approx 24 \mathrm{~m}^{-3} \text { at } 500 \mathrm{GeV} \text { beam energy } .
$$




\section{DISCLAIMER}

This report was prepared as an account of work sponsored by an agency of the United States Government. Neither the United States Government nor any agency thereof, nor any of their employees, makes any warranty, express or implied, or assumes any legal liability or responsibility for the accuracy, completeness, or usefulness of any information, apparatus, product, or process disclosed, or represents that its use would not infringe privately owned rights. Reference herein to any specific commercial product, process, or service by trade name, trademark, manufacturer, or otherwise does not necessarily constitute or imply its endorsement, recommendation, or favoring by the United States Government or any agency thereof. The views and opinions of authors expressed herein do not necessarily state or reflect those of the United States Government or any agency thereof. 


\section{DISCLAIMER}

Portions of this document may be illegible in electronic image products. Images are produced from the best available original document. 


\section{F. The $\Delta$-Values}

To determine the optimum relative spot-size increases $\Delta_{x}, \Delta_{y}$, and $\Delta_{E}$, we take $\Delta \equiv \Delta_{E}=\Delta_{y}$. Using $\beta_{x, y}^{*} \sim$ $1 / \Delta_{x, y}$ (which follows from $\xi_{x, y}^{(3)} \sim 1 / \beta_{x, y}^{*}, \beta_{D}^{x, y} \sim 1 / \beta_{x, y}^{*}$, and Eqs. (18), (19), and (20)), we find

$$
\sigma_{y}^{2} \propto \frac{1}{\Delta}\left(1+2 \Delta^{2}\right) \quad \text { and } \quad \sigma_{x}^{2} \propto \frac{1}{\Delta_{x}}\left(1+\Delta_{x}^{2}\right),
$$

from which the smallest spot size is obtained for

$$
\Delta_{y} \approx \Delta_{E} \approx \frac{1}{\sqrt{2}} \quad \text { and } \quad \Delta_{x} \approx 1 .
$$

\section{G. Vibration Tolerance and Power Supply Ripple}

Denoting the horizontal vibration amplitude of the $\mathrm{Y}$ sextupoles (this is equivalent to an orbit-change due to a vibrating quadrupole between the sextupole-pair) by $\Delta x$, we find

$$
k_{s} \beta_{D}^{y} \leq \frac{1}{5 \Delta x}
$$

allowing a maximum spot size increase of $2 \%$ due to the induced waist shift. Moreover, if we suppose that the strength of all quadrupoles in the CCY varies by a factor $\Delta k / k$ due to power supply ripple, it follows

$$
\beta_{D}^{y} \leq \frac{L}{5 \frac{\Delta k}{k} \hat{c}_{C Y}^{y}} .
$$

This has to be compared with inequality (19).

\section{OPTIMIZATION}

The minimum length of a final focus can be derived from the eight conditions (18), (19), (20), (23), (24), (26), (31) and (32). The achievable orbit stability at the second Y-sextupole regarding perturbations internal to the CCY, $\Delta x$, determines the maximum value of $k_{D} \beta_{D}^{y}$ via Eq. (31), while Eq. (18) gives a minimum value of $\beta_{D}^{x} / L$. If these two limits are inserted into Eq. (20), a lower bound on $\beta_{D}^{y} / L^{2}$ is obtained. Inequality (23) shows that the smallest value for $\eta_{D}$ allows the shortest length $L$. Ideally, therefore, we would like to choose the smallest value for $\beta_{D}^{y} / L$ on the right-hand side of (24). However, a compromise has to be made in order to keep the sextupole strength $k_{D}$ at a tolerable level. The semi-arbitrary requirement that the second term on the right-hand side contribute about $15 \%$ to the total may be a reasonable choice. Inserting the optimum value of the dispersion $\eta_{D}$, deduced from Eq. (24), into Eq. (23), the minimum length $L$ follows. It still remains to be verified whether the usually looser conditions (19), (26) and (32) are fulfilled.

As an example, from the initial design of an NLC final focus at $1 \mathrm{TeV}$, we extract $\bar{r}_{12} \approx 0.062, b \approx 1.6, \hat{c}_{C Y}^{y} \approx-24$, $\hat{c}_{C Y}^{y^{\prime \prime}} \approx-0.67, \hat{c}_{C Y}^{x^{\prime \prime}} \approx 0.12, \xi_{x}^{(3)} \approx-2000\left(\right.$ for $\beta_{x}^{*} \approx 25 \mathrm{~mm}$ ), $\xi_{y}^{(3)} \approx 15800$, and $\alpha \approx 34$. The rms-energy spread is taken to be $\delta \approx 2 \times 10^{-3}$, the horizontal normalized emittance $\epsilon_{x N} \approx 5 \mathrm{~mm} \mathrm{mrad}$, and the emittance ratio $\epsilon_{x} / \epsilon_{y} \approx 100$.

Assuming that at the second sextupole an orbit stability of $\Delta x \approx 230 \mathrm{~nm}$ can be achieved, the optimum finalfocus parameters are-obtained by the outlined procedure.
They are listed in Table I and compared with the initial and the present final focus design. The length of the final focus was shortened by about a factor of two. This was achieved by lowering the value of dispersion and beta functions at the Y-sextupoles, while increasing the sextupole strength $k_{D}$. The present design is even somewhat shorter than the estimated optimum. The reason for this is that new sextupoles, similar in spirit to those proposed by Brinkmann [4], have been added throughout the system, which locally correct the chromaticity in each module. For more details on the NLC final focus, see Ref. [5].

\section{Table I}

Initial and optimized CCY parameters for an NLC final focus system at $1 \mathrm{TeV}$ c.m. energy.

\begin{tabular}{|c|c|c|c|}
\hline \multirow{2}{*}{ Parameter } & \multicolumn{3}{|c|}{ T TeV } \\
\cline { 2 - 4 } & Initial & Optimum & Present \\
\hline \hline$\beta_{D}^{g}[\mathrm{~km}]$ & 160 & 140 & 120 \\
\hline$\eta_{D}[\mathrm{~mm}]$ & 45 & 24 & 23 \\
\hline$k_{D}\left[\mathrm{~m}^{-2}\right]$ & 2.8 & 6.4 & 7.4 \\
\hline$l_{D}[\mathrm{~m}]$ & 0.4 & 0.4 & 0.4 \\
\hline$\Delta x[\mathrm{~nm}]$ & 400 & 230 & 230 \\
\hline$\Delta k / k$ & $8 \cdot 10^{-5}$ & $6 \cdot 10^{-5}$ & $6 \cdot 10^{-5}$ \\
\hline \hline$L_{\text {tot }}[\mathrm{m}]$ & 1461 & 917 & 791 \\
\hline \hline
\end{tabular}

\section{SUMMARY}

We have derived eight scaling laws that characterize the length-dependent effects in a final focus system, and can be used as a guideline for optimization. The optimum length of the NLC final focus is primarily determined by the achievable level of orbit perturbations internal to the CCY, as measured at the second $\mathrm{Y}$-sextupole, and by the effect of synchrotron radiation in the bending magnets. Assuming an orbit stability of $\Delta x \approx 230 \mathrm{~nm}$, the initial length of the final focus design for an NLC with $1 \mathrm{TeV}$ c.m. energy was reduced by about a factor of two. The distance from the CCX to the IP is now about $800 \mathrm{~m}$. For a final focus system at a c.m. energy of $1.5 \mathrm{TeV}$, the optimum length is estimated to be about $1000 \mathrm{~m}$.

\section{References}

[1] J. Irwin, "Final Focus System Optimization," in the Proc. of "Linear Colliders. 3rd International Workshop, LC 91, Protvino, USSR," Vol. 3, V. Balakin et al. (ed.), 1991.

[2] J. Irwin, "The Application of Lie Algebra Techniques to Beam Transport Design," SLAC-PUB-5315, published in NIM A298, 460, 1990.

[3] M. Sands, "The Physics of Electron Storage Rings," SLAC-121, 1971.

[4] R. Brinkmann, "Optimization of a Final Focus System for Large Momentum Bandwidth," DESY-M-90$14,1990$.

[5] F. Zimmermann et al., "A Final Focus System for the Next Linear Collider," these proceedings, 1995. 